\title{
Report on Science Project for Probing the High-Latitude Ionosphere with Complementary Radar and Energetic Neutral Atom (ENA) Measurements
}

\author{
Daniel Martini1 ${ }^{*}$, Carl-Fredrik Enell2 ${ }^{2}$, Anita Aiko ${ }^{3}$, Thomas Ulich ${ }^{4}$, Young-Sil Kwak ${ }^{5}$ \\ ${ }^{1}$ School of Space Research, Kyung Hee University, Seoul, Korea \\ ${ }^{2}$ EISCAT Scientific Association, Kiruna, Sweden \\ ${ }^{3}$ Space Physics Group, University of Oulu, Oulu, Finland \\ ${ }^{4}$ Sodankylä Geophysical Observatory, University of Oulu, Sodankylä, Finland \\ ${ }^{5}$ Korean Astronomy and Space Science Institute (KASI), Daejeon, Korea \\ Email: ${ }^{*}$ dmartini@khu.ac.kr
}

Received 25 March 2014; revised 22 April 2014; accepted 7 May 2014

Copyright (C) 2014 by authors and Scientific Research Publishing Inc.

This work is licensed under the Creative Commons Attribution International License (CC BY).

http://creativecommons.org/licenses/by/4.0/

(c) (i) Open Access

\section{Abstract}

Vast magnetospheric regions are mapped along the field lines to the narrow latitudinal band of the polar/auroral regions. Therefore monitoring of solar wind energy dissipation into the ionosphere at auroral latitudes gives unique opportunities to study geomagnetic disturbances in their complexity from a relatively well-localized vantage point. Here we introduce and present the current state of a recently proposed science project for coordinated monitoring of high-latitude activity with the EISCAT (European Incoherent Scatter) radar array supported by ground-based magnetometer and optical data and ENA (Energetic Neutral Atom) observation from the CINEMA (CubeSat for Ions, Neutrals, Electrons and Magnetic field) satellite system.

\section{Keywords}

Energetic Neutral Atoms, Incoherent Scatter Radar, Satellite Observation, Ionsphere, Geomagnetic Activity

\footnotetext{
"Corresponding author.
}

How to cite this paper: Martini, D., et al. (2014) Report on Science Project for Probing the High-Latitude lonosphere with Complementary Radar and Energetic Neutral Atom (ENA) Measurements. International Journal of Geosciences, 5, 607-610. http://dx.doi.org/10.4236/ijg.2014.56055 


\section{Introduction}

Physical processes in the vast cavity of Earth's magnetosphere have been studied by an ever increasing number of satellite missions. While single satellite in situ measurements provide us invaluable information about magnetospheric processes, they raise the critical problem of separation of spatial and temporal evolution of the observed phenomena. It cannot be deduced solely by single-point data whether any detected change reflects temporal evolution of a single feature, or if consecutive measurement points probe different parts in space of a static phenomenon. To resolve this problem, multi-point measurements are required. A solution is to use remote imaging techniques that map a given process of interest as a whole instead of the mosaic-like in situ probing.

\section{Energetic Neutral Atom (ENA) Observations}

\subsection{The ENA Technique}

ENA (Energetic Neutral Atoms) are produced when energetic ions collide and exchange charge with the cold and dense neutral upper atmosphere, also called the geocorona. When charge exchange has taken place, the formerly charged ion becomes a neutral atom, de-coupled from the magnetic field, and thus continues its trajectory along an unperturbed straight line, carrying along such important information as its original energy and pitch angle virtually unchanged ([1], and references therein). In this way, ENA imaging is highly analogous with visible light (photon) imaging, with the geocorona serving as a background screen. Detecting ENA gives the only means for global imaging of the various ion populations of the magnetosphere, and therefore provides the means to monitor the corresponding processes as whole and remotely. Using more than one ENA imaging satellite maximizes the chance for separating spatial and temporal developments with high credence.

\subsection{CubeSat for Ions, Neutrals, Electrons and Magnetic Field (CINEMA)}

The School of Space Research, Kyung Hee University, South Korea, in collaboration with the Space Sciences Laboratory, University of California at Berkeley, USA and Imperial College, London, UK, recently developed two identical CINEMA (CubeSat for Ions, Neutrals, Electrons and Magnetic field) satellites, designated for stereo imaging. Each satellite consists of three standard CubeSat units that incorporate the operating and avionics electronics and a scientific payload with a total mass of about $3 \mathrm{~kg}$ (see Figure 1). The scientific payload includes a three-axis magnetoresistive magnetometer with $0.25 \mathrm{nT}$ sensitivity on a deployable boom developed by the Imperial College, and as main payload the ENA detector of STEIN (Suprathermal Electrons, Ions and Neutrals), also depicted in Figure 2.

The same STEIN-type detector (among other instruments) will be used on board of the upcoming Solar Orbiter sun observing satellite of the ESA (European Space Agency) to be launched in 2017. Therefore our micro

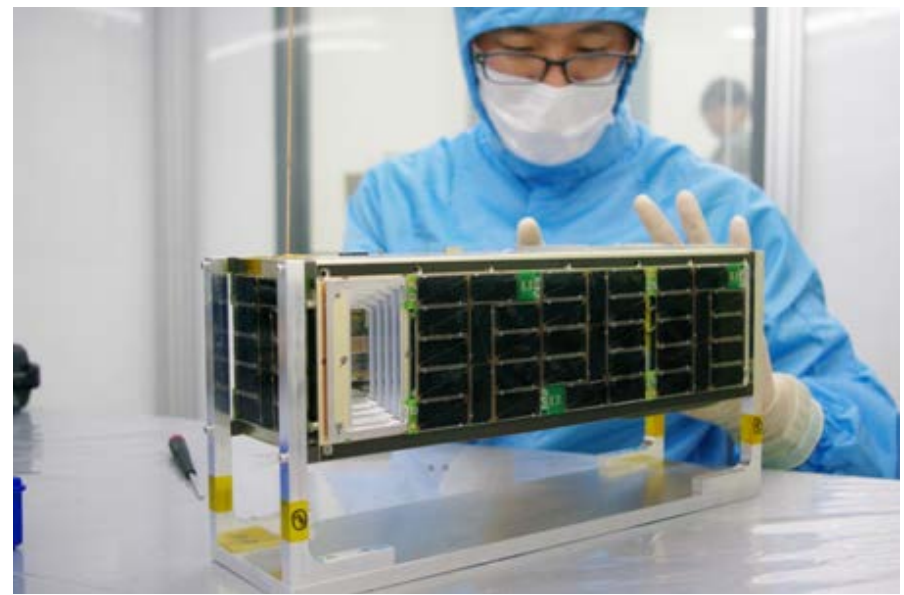

Figure 1. One of the flight model of CINEMA satellite-pair. STEIN housing with collimator appears in the forefront (note, the collimator is yet uncoated). Solar panels with thermometers (green squares) are also visible. 


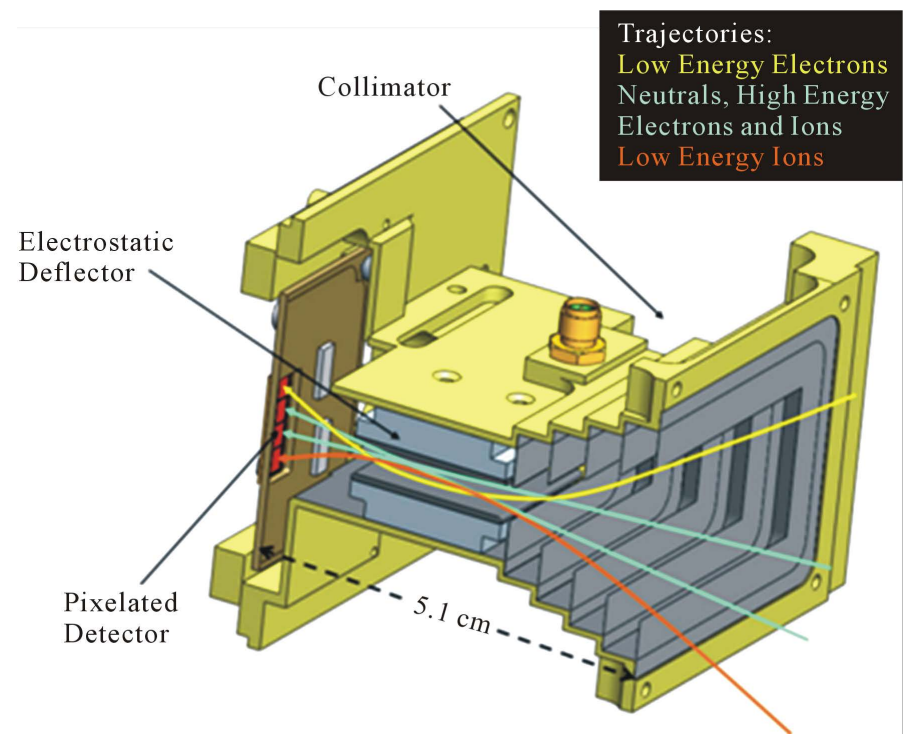

Figure 2. Cutaway view of the STEIN detector together with the electrostatic deflector and collimator. The deflected paths of electrons/ions and the straight paths of neutrals and energetic charged particles are also indicated on the picture.

satellite mission may be viewed as a test run of a detector that may well be used frequently in future missions of similar kind due to its small physical dimensions and power requirement.

Unlike standard electrostatic analyzers used to detect charged particles, STEIN [2] uses a silicon diode detec-

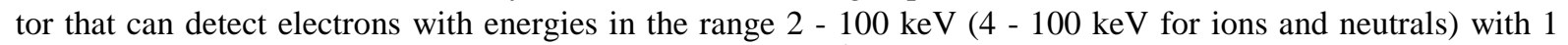
$\mathrm{keV}$ FWHM resolution and a geometrical factor of $0.02 \mathrm{~cm}^{2} \mathrm{sr}$. This solid state sensor is equipped with an electrostatic deflector plate-pair and the instrument has a uniquely low mass $(<500 \mathrm{~g})$ and power consumption $(0.5$ W). The symmetric arrangement of the deflection system allows detecting all electrons, ions, and neutrals with one single detector. At a given applied deflection voltage ions and electrons are deflected to the opposite extreme pixels, while neutrals excite the central pixels. Laboratory tests show that the maximum deflection voltage of $4 \mathrm{kV}$ allows charge separation up to about $20 \mathrm{keV}$ incident energy, above which STEIN operates as an in situ particle detector without charge separation. STEIN is also equipped with a 100 -fold mechanical attenuator for cases when particle flux is extremely high.

The CINEMA satellite pair was successfully launched on the 22nd November, 2013 into a pre-defined high inclination (93 degrees) low altitude ( $\sim 800 \mathrm{~km})$ sun-synchronous spin stabilized orbit (4 RPM). The orbit and spin axis were designed so that STEIN may detect both direct ENA emissions, e.g. from the ring current, and the trapped and precipitating particles at auroral latitudes. Although equatorial ion density is much larger than that in the precipitation region, the geocorona is much denser at precipitation altitudes, resulting in an overall stronger flux from 500 - $800 \mathrm{~km}$ altitudes in the auroral zone, i.e., from the auroral top-side ionosphere.

Since the Earth's magnetic field connects the ionosphere at high latitudes to the magnetosphere through particle precipitation, coordinated ground-based high-latitude observations are an intrinsic method to better understand the solar wind energy transfer into the coupled ionosphere/magnetosphere system. The typical ENA source region at this latitude and CINEMA's orbit allow incorporating data from a number of ground-based instruments for a comprehensive study of the complex phenomena of magnetic storms and substorms.

\section{The Coordinated EISCAT and STEIN ("EINSTEIN") Measurement Project}

The incoherent scatter radar technique provides important parameters of the ionospheric plasma, such as Ne (electron density), $\mathrm{Te} / \mathrm{Ti}$ (ion and electron temperatures), and $\mathrm{Vi}$ (line of sight ion velocity), up to an altitude of about $1000 \mathrm{~km}$. Therefore, combining simultaneous distant ENA and radar observations, it is possible to map the energy dissipation mechanisms all the way from the magnetosphere (through distant ENA imaging of ring current, or tail acceleration regions) down to the ionosphere (via radar observations and direct precipitating par- 
ticle detection by STEIN). For this purpose, researchers from four institutions: 1) Kyung Hee University, Korea, 2) EISCAT Scientific Association, Sweden, 3) University of Oulu, Finland, and 4) Korean Astronomy and Space Science Institute, Korea, have proposed the coordinated EISCAT (European Incoherent Scatter) radar and STEIN measurement project ("EINSTEIN"). With the additional use of ground-based magnetometer and optical data in the vicinity of the radar site, our project has the following science objectives: a) improve our current understanding of how the dramatic sudden eruptions of substorms are initiated by closely monitoring the phenomenon from its initiation in the near-Earth magnetotail to the final dissipation of energy as low as the E-region ionosphere at altitudes of about $90 \mathrm{~km}$. Furthermore, b) we also hope to gain a better understanding of how auroral and polar ion upflows are triggered by precipitation and what their role is in magnetosphric enrichment with ionospheric ions.

In December 2013 and January 2014, extensive initial tests were conducted in order to find the optimal radar experiment settings that best address our above science objectives. Accordingly, the EISCAT Svalbard Radar (ESR) at Longyearbyen, Svalbard, operated by the EISCAT Scientific Association, will be coordinated with CINEMA fly bys as follows; the $42 \mathrm{~m}$ dish (fixed in field aligned position) will detect precipitation, while the 32 $\mathrm{m}$ steerable dish will be pointed southward along the magnetic meridian with a low elevation of about 30 degrees. The latter positioning allows monitoring the auroral oval dynamics, as well as estimating $\mathrm{E}_{\mathrm{Ew}}$ (east-west component of the local electric field, E) in addition to the above listed fundamental plasma parameters.

The next stage of actual coordinated measurements is expected to begin shortly, after the initial calibration procedures of the on-board instruments and the two-way data transfer with the CINEMA satellites are successfully established and secured. We hope to share the first quantitative results of our joint effort during this year, 2014.

\section{Acknowledgements}

This work has been supported by the BK21 Plus program through the National Research Foundation (NRF) funded by the Ministry of Education of Korea. D. Martini thanks Ingemar Haggström at EISCAT Scientific Association for the valuable personal discussions.

\section{References}

[1] Brandt, C.P., Mitchell, D.G., Roelof, E.C., Krimigis, S.M., Paranicas, C.P., Mauk, B.H., Saur, J. and DeMajistre, R. (2005) ENA Imaging: Seeing the Invisible. Johns Hopkins APL Technical Digest, 26.

[2] Glaser, D.L., Halekas, J.S., Turin, P., Curtis, D.W., Larson, D.E., McBride, S.E. and Lin, R.P. (2009) STEIN (SupraThermal Electrons, Ions and Neutrals), a New Particle Detection Instrument for Space Weather Research with CubeSats, SSC09-III-1. 23rd Annual AIAA/USU Conference on Small Satellites. 